\title{
A PROBLEM OF RAMANUJAN, ERDŐS AND KÁTAI ON THE ITERATED DIVISOR FUNCTION
}

\author{
YVONNE BUTTKEWITZ, CHRISTIAN ELSHOLTZ, KEVIN FORD, AND JAN-CHRISTOPH SCHLAGE-PUCHTA
}

\begin{abstract}
We determine asymptotically the maximal order of $\log d(d(n))$, where $d(n)$ is the number of positive divisors of $n$. This solves a problem first put forth by Ramanujan in 1915.
\end{abstract}

\section{Introduction}

Let $d(n)$ denote the number of positive divisors of an integer $n$. The extreme large values of $d(n)$ were studied by Wigert [10], (see also [4, Theorem 432]). Wigert proved that

$$
m_{1}(x):=\max _{n \leqslant x} \log d(n) \sim(\log 2) \frac{\log x}{\log _{2} x} .
$$

Here $\log _{k} x$ denotes the $k$-th iterate of the logarithm. The lower bound comes from considering integers of the form $N_{k}=p_{1} \cdots p_{k}$, where $p_{j}$ denotes the $j$ th smallest prime. Here $d\left(N_{k}\right)=2^{k}$, while $\log N_{k} \sim k \log k$ by the prime number theorem. In his seminal 1915 paper on highly composite numbers [7], Ramanujan gave a more precise asymptotic for $m_{1}(x)$. At the very end of his paper, Ramanujan posed the problem of finding the extreme large values of $d(d(n))$. By considering integers of the form

$$
2^{1} \cdot 3^{2} \cdot 5^{4} \cdots p_{k}^{p_{k}-1}
$$

Ramanujan showed that

$$
m_{2}(x):=\max _{n \leqslant x} \log d(d(n)) \geqslant(\sqrt{2} \log 4+o(1)) \frac{\sqrt{\log x}}{\log _{2} x} .
$$

The problem of finding the order of $m_{2}(x)$ has been mentioned in Erdős [1], Ivić [5], and has been mentioned by Ivić in problem sessions in Ottawa [6] and Oberwolfach.

Erdôs and Kátai [3] showed $m_{2}(x)=(\log x)^{1 / 2}\left(\log _{2} x\right)^{O(1)}$ (see (4.1) on p. 270 of [3]). Twenty years later Erdős and Ivić [2] improved the upper bound to

$$
m_{2}(x) \ll\left(\frac{\log x \log _{2} x}{\log _{3} x}\right)^{1 / 2} .
$$

Smati [8, 9] gave a further improvement

$$
m_{2}(x) \ll \sqrt{\log x}
$$

the best estimate known to date. Constructions similar to Ramanujan's seem rather natural, and one might expect that $m_{2}(x) \ll \frac{\sqrt{\log x}}{\log _{2} x}$. This is indeed the case, as we now show. More precisely, we prove an asymptotic formula for $m_{2}(x)$ with an error term.

Theorem 1. We have

$$
m_{2}(x)=\frac{\sqrt{\log x}}{\log _{2} x}\left(c+O\left(\frac{\log _{3} x}{\log _{2} x}\right)\right),
$$

Date: May 10, 2018.

The work of Y. B. and C. E. was supported by the German Research Council (DFG-Grant-Number BU2488/1-3). K. F. was supported by National Science Foundation grant DMS-0901339. 
where

$$
c=\left(8 \sum_{j=1}^{\infty} \log ^{2}(1+1 / j)\right)^{1 / 2}=2.7959802335 \ldots
$$

In particular, Theorem 1 implies that

$$
\limsup _{n \rightarrow \infty} \frac{\log d(d(n)) \log _{2} n}{\sqrt{\log n}}=c .
$$

Ramanujan's examples (1.1) are seen to be suboptimal with respect to the constant $c$, since $\sqrt{2} \log 4=$ $1.9605 \ldots$

There is a closely related problem, to estimate the extreme values of $\omega(d(n))$, where $\omega(n)$ is the number of distinct prime factors of $n$. In fact, both Erdős and Ivić [2] and Smati [9] obtained upper bounds for $d(d(n))$ by first bounding $\omega(d(n))$ and then using the elementary inequality $\log d(m) \ll\left(\log _{2} m\right) \omega(m)$ (see, e.g., Lemme 3.3 of [8] or Lemma 3.2 below). For this problem, Ramanujan's examples (1.1) are essentially optimal, providing the true order and constant in the asymptotic for $w(x)=\max _{n \leqslant x} \omega(d(n))$.

Theorem 2. We have

$$
w(x)=\frac{\sqrt{\log x}}{\log _{2} x}\left(\sqrt{8}+O\left(\frac{\log _{3} x}{\log _{2} x}\right)\right),
$$

Previously, Erdôs and Ivić [2] had shown

$$
w(x) \ll\left(\frac{\log x \log _{3} x}{\log _{2} x}\right)^{1 / 2},
$$

and later Smati [8] found the true order $w(x) \ll \frac{\sqrt{\log x}}{\log _{2} x}$.

\section{The lower bound in Theorem 1}

Notation and basic prime number estimates. Throughout, we make use of the asymptotic

$$
p_{j}=j\left(\log j+\log _{2} j+O(1)\right),
$$

which is a simple consequence of the prime number theorem with error term $\pi(x)=\frac{x}{\log x}+O\left(\frac{x}{\log ^{2} x}\right)$. Here $\pi(x)$ is the number of primes which are $\leqslant x$. We also denote by $\Omega(n)$ the number of prime power divisors of $n$.

Proof of the lower bound in Theorem 1 Let $x$ be large and define $\varepsilon=10 \frac{\log _{3} x}{\log _{2} x}$. Let

$$
t=\left\lfloor\left(\frac{8 \log 2}{c}-\varepsilon\right) \frac{\sqrt{\log x}}{\log _{2} x}\right\rfloor, \quad a_{i}=\left\lfloor\frac{1}{2^{i / t}-1}\right\rfloor \quad(1 \leqslant i \leqslant t),
$$

and let

$$
n=\left(p_{1} \cdots p_{a_{1}}\right)^{p_{1}-1}\left(p_{a_{1}+1} \cdots p_{a_{1}+a_{2}}\right)^{p_{2}-1} \cdots\left(p_{a_{1}+\cdots+a_{t-1}+1} \cdots p_{a_{1}+\cdots+a_{t}}\right)^{p_{t}-1} .
$$

The Taylor expansion of $\exp \left(\frac{\log 2}{t}\right)$ shows that $a_{1}=\left\lfloor\left(2^{1 / t}-1\right)^{-1}\right\rfloor=t / \log 2+O(1)$. By (2.2), for every positive integer $j$, there are $y_{j}:=\left\lfloor\frac{\log (1+1 / j)}{\log 2} t\right\rfloor$ indices $i$ with $a_{i} \geqslant j$. Also, $a_{1}+\cdots+a_{t} \ll t \log t$. Using (2.1), we have $\log p_{a_{1}+\cdots+a_{i}} \leqslant \log t+2 \log _{2} t+O(1)$, hence

$$
\log n \leqslant \sum_{i=1}^{t} a_{i}\left(p_{i}-1\right) \log p_{a_{1}+\cdots+a_{i}} \leqslant\left(\log ^{2} t+3\left(\log _{2} t\right) \log t+O(\log t)\right) \sum_{i=1}^{t} i a_{i} .
$$


From $y_{j}=O(t / j)$ and the definition of $c$ we obtain

$$
\begin{aligned}
\sum_{i=1}^{t} i a_{i}=\sum_{j \leqslant a_{1}} \frac{y_{j}\left(y_{j}+1\right)}{2} & =\frac{1}{2} \sum_{j=1}^{\infty}\left(\frac{\log (1+1 / j)}{\log 2}\right)^{2} t^{2}+O(t \log t) \\
& =\frac{c^{2}}{16(\log 2)^{2}} t^{2}+O(t \log t) .
\end{aligned}
$$

From the definition of $t, \log t=\frac{1}{2} \log _{2} x-\log _{3} x+O(1)$ and $\log _{2} t=\log _{3} x+O(1)$. Thus,

$$
\log n \leqslant\left(1+\frac{2 \log _{3} x+O(1)}{\log _{2} x}\right)\left(1-\frac{c \varepsilon}{8 \log 2}\right)^{2} \log x .
$$

Hence, if $x$ is large enough, then $n \leqslant x$. From the definition of $n$ above, we have $d(n)=p_{1}^{a_{1}} \cdots p_{t}^{a_{t}}$. Therefore,

$$
\begin{aligned}
\log m_{2}(x) \geqslant \log d(d(n)) & =\sum_{i=1}^{t} \log \left(a_{i}+1\right)=\sum_{j \geqslant 1}\left(y_{j}-y_{j+1}\right) \log (j+1)=\sum_{j \geqslant 1} y_{j} \log (1+1 / j) \\
& =\sum_{j \leqslant a_{1}}\left(\frac{\log ^{2}(1+1 / j)}{\log 2} t+O(1 / j)\right) \\
& =\frac{c^{2}}{8 \log 2} t+O(\log t) \\
& =\frac{\sqrt{\log x}}{\log _{2} x}\left(c+O\left(\frac{\log _{3} x}{\log _{2} x}\right)\right)
\end{aligned}
$$

\section{Proof of the upper bound in Theorem 1}

Lemma 3.1. Let $m_{N}=\min \{m: d(m)=N\}$ and write $m_{N}=p_{1}^{\alpha_{1}} \cdots p_{r}^{\alpha_{r}}$. We have

(i) $\alpha_{1} \geqslant \cdots \geqslant \alpha_{r}$,

(ii) $N^{\prime} \mid N$ implies $m_{N^{\prime}} \leqslant m_{N}$,

(iii) for each integer $k \geqslant 1$, if $p_{j}>p_{r+1}^{1 / 2^{k}}$, then $\Omega\left(\alpha_{j}+1\right) \leqslant k$.

Remark 1. Using (2.1) and taking $k=1$, we see from (iii) that if $r$ is large, then $\alpha_{j}+1$ is prime for $\sqrt{r}<j \leqslant r$. Also, by (iii), $\Omega\left(\alpha_{j}+1\right) \ll \log _{2} r$ for all $j$.

Proof. (i) This is trivial and was observed by Ramanujan [7, (32)].

(ii) If $N^{\prime} \mid N$, we can find $\alpha_{j}^{\prime} \leqslant \alpha_{j}$ for each $j$ such that $N^{\prime}=\left(\alpha_{1}^{\prime}+1\right) \cdots\left(\alpha_{r}^{\prime}+1\right)$, and clearly $m_{N^{\prime}} \leqslant$ $p_{1}^{\alpha_{1}^{\prime}} \cdots p_{r}^{\alpha_{r}^{\prime}} \leqslant m_{N}$.

(iii) If $p_{j}>p_{r+1}^{1 / 2^{k}}$ and $\Omega\left(\alpha_{j}+1\right)>k$, then there are integers $a, b$ with $\alpha_{j}+1=a b, a \geqslant 2$ and $b \geqslant 2^{k}$. Letting

$$
m^{*}=p_{j}^{b-1} p_{r+1}^{a-1} \prod_{i \neq j} p_{i}^{\alpha_{i}}
$$

we see that $d\left(m^{*}\right)=d\left(m_{N}\right)=N$, but

$$
\frac{m^{*}}{m_{N}}=p_{j}^{b-1-\alpha_{j}} p_{r+1}^{a-1}=\left(p_{j}^{-b} p_{r+1}\right)^{a-1}<1,
$$

a contradiction. 
Lemma 3.2. For every $\varepsilon>0$, and for $\omega(n)=s \geqslant 2$ we have

$$
d(n) \ll_{\varepsilon}\left(\frac{(2+\varepsilon) \log n}{s \log s}\right)^{s} .
$$

Proof. Write the prime factorization of $n$ as $n=q_{1}^{a_{1}} \cdots q_{s}^{a_{s}}$, where $q_{1}<\cdots<q_{s}$. Using the arithmetic mean - geometric mean inequality and that $q_{i} \geqslant p_{i}$, we have

$$
d(n) \leqslant \prod_{i=1}^{s}\left(2 a_{i}\right) \leqslant 2^{s} \prod_{i=1}^{s}\left(a_{i} \log q_{i}\right) \prod_{i=1}^{s}\left(\log p_{i}\right)^{-1} \leqslant\left(\frac{2 \log n}{s}\right)^{s} \frac{(\log s)^{\pi(s)-s}}{\log 2},
$$

the last inequality coming from excluding factors corresponding to $3 \leqslant p_{i}<s$. Finally, the prime number theorem implies $(\log s)^{\pi(s)} \leqslant(\log s)^{O(s / \log s)} \ll_{\varepsilon}(1+\varepsilon / 2)^{s}$.

Remark. Lemma 3.2 is fairly sharp. For example, from the inequality $s=\omega(n) \leqslant(1+o(1)) \frac{\log n}{\log _{2} n}$, and the observation that $m_{1}(x)$ is monotonically increasing, we immediately obtain Wigert's upper bound for $\log d(n)$.

The following is the key lemma, which explains the constant $c$.

Lemma 3.3. Let $a_{1}, \ldots, a_{t}$ be positive integers.

(a) we have

$$
\sum_{i=1}^{t} \log \left(a_{i}+1\right) \leqslant \frac{c}{2}\left(\sum_{i=1}^{t} i a_{i}\right)^{1 / 2}
$$

Moreover, the constant $c / 2$ is best possible.

(b) If $a_{i} \geqslant A$ for all $i$, where $A$ is a positive integer, then

$$
\sum_{i=1}^{t} \log \left(a_{i}+1\right) \leqslant\left(\frac{1+\log ^{2}(A+1)}{A} \sum_{i=1}^{t} i a_{i}\right)^{1 / 2} .
$$

Proof. (a) Without loss of generality, suppose $a_{1} \geqslant \cdots \geqslant a_{t}$. Let $y_{j}=\#\left\{i: a_{i} \geqslant j\right\}$. Then

$$
\sum_{i=1}^{t} i a_{i}=\sum_{j \geqslant 1} \frac{y_{j}\left(y_{j}+1\right)}{2} \geqslant \frac{1}{2} \sum_{j \geqslant 1} y_{j}^{2}
$$

By partial summation and the Cauchy-Schwarz inequality,

$$
\begin{aligned}
\sum_{i=1}^{t} \log \left(a_{i}+1\right)=\sum_{j \geqslant 1}\left(y_{j}-y_{j+1}\right) \log (j+1) & =\sum_{j \geqslant 1} y_{j} \log (1+1 / j) \\
& \leqslant\left(\sum_{j \geqslant 1} y_{j}^{2}\right)^{1 / 2}\left(\frac{c^{2}}{8}\right)^{1 / 2} .
\end{aligned}
$$

Moreover, the inequality in (3.1) is an equality if and only if for some real $Y, y_{j}=Y \log (1+1 / j)$ for every $j$. As the $y_{j}$ are integers, this cannot happen. However, we can come very close to equality in (3.1) by taking $t$ large and choosing the $a_{i}$ by (2.2), so that $y_{j}=\left\lfloor\frac{\log (1+1 / j)}{\log 2} t\right\rfloor$. By (2.3) and (2.4), we have in this case

$$
\sum_{i=1}^{t} \log \left(a_{i}+1\right)=\frac{c^{2}}{8 \log 2} t+O(\log t), \quad \sum_{i=1}^{t} i a_{i}=\frac{c^{2}}{16(\log 2)^{2}} t^{2}+O(t \log t)
$$

whence

$$
\sum_{i=1}^{t} \log \left(a_{i}+1\right)=\frac{c}{2}\left(1+O\left(\frac{\log t}{t}\right)\right)\left(\sum_{i=1}^{t} i a_{i}\right)^{1 / 2}
$$


(b) Observe that $y_{1}=y_{2}=\cdots=y_{A}$. Arguing similarly to 3.1 , we obtain

$$
\begin{aligned}
\sum_{i=1}^{t} \log \left(a_{i}+1\right) & =\frac{\log (A+1)}{A}\left(y_{1}+\cdots+y_{A}\right)+\sum_{j>A} y_{j} \log (1+1 / j) \\
& \leqslant\left(\sum_{j \geqslant 1} y_{j}^{2}\right)^{1 / 2}\left(A\left(\frac{\log (A+1)}{A}\right)^{2}+\sum_{j>A} \log ^{2}(1+1 / j)\right)^{1 / 2} .
\end{aligned}
$$

Observing that $\log (1+1 / j)<1 / j$ and $\sum_{j>A} 1 / j^{2}<1 / A$, we obtain (b).

The next lemma is trivial.

Lemma 3.4. For any positive integer $m, m \geqslant \sum_{p \mid m} p$.

Proof of Theorem $\square$ upper bound. Let $n$ be large, let $N=d(n)$ and factor $N=N^{\prime} N^{\prime \prime}$, where

$$
N^{\prime}=u_{1}^{b_{1}} \cdots u_{w}^{b_{w}}, \quad N^{\prime \prime}=q_{1}^{a_{1}} \cdots q_{s}^{a_{s}},
$$

where $u_{1}<\cdots<u_{w}, q_{1}<\cdots<q_{s}$ are primes, $b_{i}>\left(\log _{2} n\right)^{6}$ for every $i$ and $a_{i} \leqslant\left(\log _{2} n\right)^{6}$ for every $i$.

Write $m_{N^{\prime}}=p_{1}^{\beta_{1}} \cdots p_{h}^{\beta_{h}}$. By Lemma3.1(ii), $m_{N^{\prime}} \leqslant m_{N} \leqslant n$, so that $h \ll \log n$. By Lemma3.1(iii), $\Omega\left(\beta_{i}+1\right) \ll \log _{2} h \ll \log _{3} n$ for every $i$. Since $d\left(m_{N^{\prime}}\right)=\left(\beta_{1}+1\right) \cdots\left(\beta_{h}+1\right)=N^{\prime}$, for each $j \leqslant h$ there are $\gg \frac{b_{j}}{\log _{3} n}$ values of $i$ for which $u_{j} \mid\left(\beta_{i}+1\right)$. Thus, by Lemma 3.4

$$
\begin{aligned}
\log n \geqslant \log m_{N^{\prime}} \geqslant(\log 2) \sum_{i=1}^{h} \beta_{i} & \geqslant \frac{\log 2}{2} \sum_{i=1}^{h}\left(\beta_{i}+1\right) \\
& \geqslant \frac{\log 2}{2} \sum_{i=1}^{h} \sum_{p \mid\left(\beta_{i}+1\right)} p \gg \sum_{j=1}^{w} u_{j} \frac{b_{j}}{\log _{3} n} \geqslant \frac{1}{\log _{3} n} \sum_{j=1}^{w} j b_{j} .
\end{aligned}
$$

Combining this estimate with Lemma 3.3 (b) with $A=\left(\log _{2} n\right)^{6}$ gives

$$
\log d\left(N^{\prime}\right)=\sum_{j=1}^{w} \log \left(b_{j}+1\right) \ll \frac{\log _{3} n}{\left(\log _{2} n\right)^{3}}\left(\sum_{j=1}^{w} j b_{j}\right)^{1 / 2} \ll \frac{(\log n)^{1 / 2}\left(\log _{3} n\right)^{3 / 2}}{\left(\log _{2} n\right)^{3}} .
$$

Next, we bound $d\left(N^{\prime \prime}\right)$.

Case 1) If $s \leqslant \frac{(\log n)^{1 / 2}}{\left(\log _{2} n\right)^{3}}$, Lemma 3.2 implies that $\log d\left(N^{\prime \prime}\right) \ll \frac{(\log n)^{1 / 2}}{\left(\log _{2} n\right)^{2}}$.

Case 2) Now suppose that $s>\frac{(\log n)^{1 / 2}}{\left(\log _{2} n\right)^{3}}$. Write $m_{N^{\prime \prime}}=p_{1}^{\alpha_{1}} \cdots p_{r}^{\alpha_{r}}$. By Lemma3.1(iii),

$$
r \leqslant \Omega\left(N^{\prime \prime}\right)=\sum_{j=1}^{s} a_{j}=\sum_{i=1}^{r} \Omega\left(\alpha_{i}+1\right) \leqslant r+\sum_{k \geqslant 2} \pi\left(p_{r+1}^{1 / 2^{k}}\right)=r+O\left((r / \log r)^{1 / 2}\right) .
$$

In particular, $r+O\left((r / \log r)^{1 / 2}\right) \geqslant a_{1}+\cdots+a_{s} \geqslant s$, so $r \gg s>\frac{(\log n)^{1 / 2}}{\left(\log _{2} n\right)^{3}}$. Thus, for large enough $n$, $a_{1}+\cdots+a_{s} \leqslant r+\sqrt{r}$. Also by Lemma 3.1 (iii), $\alpha_{j}+1$ is prime for $j>\sqrt{r}$. Let $\varepsilon=20 \frac{\log _{3} n}{\log _{2} n}$. By the lower bound on $s$, and using $a_{i} \leqslant\left(\log _{2} n\right)^{6}$,

$$
\sum_{j>s-s^{1-\varepsilon}} a_{j} \geqslant s^{1-\varepsilon} \geqslant 2\left(s\left(\log _{2} n\right)^{6}\right)^{1 / 2} \geqslant 2\left(\Omega\left(N^{\prime \prime}\right)\right)^{1 / 2} \geqslant 2 \sqrt{r}
$$

hence, using (3.3),

$$
\sum_{j \leqslant s-s^{1-\varepsilon}} a_{j} \leqslant \Omega\left(N^{\prime \prime}\right)-2 \sqrt{r} \leqslant r-\sqrt{r}
$$


Using Lemma 3.1 (i), $\alpha_{i}+1=q_{1}$ for $r-a_{1}<i \leqslant r$, and similarly for each $j \leqslant s-s^{1-\varepsilon}, \alpha_{i}+1=q_{j}$ for $r-\left(a_{1}+\cdots+a_{j}\right)<i \leqslant r-\left(a_{1}+\cdots+a_{j-1}\right)$. We obtain

$$
\begin{aligned}
\log m_{N^{\prime \prime}} & \geqslant \sum_{\sqrt{r}<i \leqslant r} \alpha_{i} \log p_{i} \geqslant \sum_{j \leqslant s-s^{1-\varepsilon}}\left(q_{j}-1\right) \sum_{m=r-\left(a_{1}+\cdots+a_{j}\right)+1}^{r-\left(a_{1}+\cdots+a_{j-1}\right)} \log p_{m} \\
& \geqslant \sum_{j \leqslant s-s^{1-\varepsilon}}\left(p_{j}-1\right) a_{j} \log \left(r-\left(a_{1}+\cdots+a_{j}\right)\right) .
\end{aligned}
$$

By (3.4), uniformly for $j \leqslant s-s^{1-\varepsilon}$ we have

$$
r-\left(a_{1}+\cdots+a_{j}\right)=r-\Omega\left(N^{\prime \prime}\right)+a_{j+1}+\cdots+a_{s} \geqslant s-j-\sqrt{r} \geqslant \frac{1}{2} s^{1-\varepsilon} .
$$

Using (2.1), $p_{j} \geqslant j \log j+1$ for large $j$. Hence, by Lemma 3.1(ii),

$$
\begin{aligned}
\log n \geqslant \log m_{N^{\prime \prime}} & \geqslant \sum_{s^{1-\varepsilon} \leqslant j \leqslant s-s^{1-\varepsilon}}(j \log j) a_{j}\left(\log s+O\left(\log _{3} n\right)\right) \\
& \geqslant(1+O(\varepsilon)) \frac{\left(\log _{2} n\right)^{2}}{4} \sum_{s^{1-\varepsilon \leqslant j \leqslant s-s^{1-\varepsilon}}} j a_{j} .
\end{aligned}
$$

By the definition of $\varepsilon, s^{\varepsilon} \gg\left(\log _{2} n\right)^{9}$. Also, trivially $\sum_{j=1}^{s} j a_{j} \geqslant 1+2+\cdots+s \geqslant \frac{1}{2} s^{2}$. Recalling that $a_{j} \leqslant\left(\log _{2} n\right)^{6}$ for every $j$, we have

$$
\begin{aligned}
\sum_{s^{1-\varepsilon} \leqslant j \leqslant s-s^{1-\varepsilon}} j a_{j} & =\sum_{j=1}^{s} j a_{j}+O\left(s^{2-\varepsilon}\left(\log _{2} n\right)^{6}\right)=\sum_{j=1}^{s} j a_{j}+O\left(s^{2}\left(\log _{2} n\right)^{-3}\right) \\
& =\left(1+O\left(1 / \log _{2} n\right)\right) \sum_{j=1}^{s} j a_{j} .
\end{aligned}
$$

Combining the last two inequalities gives

$$
\log n \geqslant\left(1+O\left(\frac{\log _{3} n}{\log _{2} n}\right)\right) \frac{\left(\log _{2} n\right)^{2}}{4} \sum_{j=1}^{s} j a_{j} .
$$

Applying Lemma 3.3 (a), we conclude that

$$
\log d\left(N^{\prime \prime}\right)=\sum_{j=1}^{s} \log \left(a_{j}+1\right) \leqslant \frac{c}{2}\left(\sum_{j=1}^{s} j a_{j}\right)^{1 / 2} \leqslant c \frac{\sqrt{\log n}}{\log _{2} n}\left(1+O\left(\frac{\log _{3} n}{\log _{2} n}\right)\right) .
$$

Recall that we have a smaller upper bound for $\log d\left(N^{\prime \prime}\right)$ in case 1). Finally, using $d(d(n))=d\left(N^{\prime}\right) d\left(N^{\prime \prime}\right)$ and combining (3.2) and (3.5), we obtain the desired upper bound for $d(d(n))$.

\section{Proof of Theorem 2}

Proof of Theorem 2 For the lower bound, let $x$ be large and put $n=\prod_{i=1}^{s} p_{i}^{p_{i}-1}$, where $s$ is the largest integer such that $n \leqslant x$. Recall that $p_{j}$ is the $j$-th smallest prime. Then $d(n)=\prod_{i=1}^{s} p_{i}$, thus $\omega(d(n))=s$. By (2.1),

$$
\log n=\sum_{i=1}^{s}\left(p_{i}-1\right) \log p_{i}=\sum_{i=1}^{s} i \log ^{2} i+O\left(i \log i \log _{2} i\right)=\frac{1}{2} s^{2} \log ^{2} s+O\left(s^{2} \log s \log _{2} s\right) .
$$


Solving for $s$ gives $s=\frac{\sqrt{8 \log n}}{\log _{2} n}+O\left(\frac{\sqrt{\log n} \log _{3} n}{\log _{2}^{2} n}\right)$. We now prove a lower bound on $n$. Since $p_{s+1} \sim$ $s \log s \sim \sqrt{2 \log n} \ll \sqrt{\log x}$ by $(2.1)$, we have

$$
x \geqslant n \geqslant x p_{s+1}^{-p_{s+1}}=x \exp \left(-O\left(\sqrt{\log x} \log _{2} x\right)\right) .
$$

That is, $\log n=\log x+O\left(\sqrt{\log x} \log _{2} x\right)$. Therefore, $s=\frac{\sqrt{8 \log x}}{\log _{2} x}+O\left(\frac{\sqrt{\log x} \log _{3} x}{\log _{2}^{2} x}\right)$.

Now let $n$ be a large, positive integer factored as $n=n_{1} n_{2}, n_{1}=\prod_{i=1}^{r} q_{i}^{a_{i}}, n_{2}=\prod_{i=1}^{r^{\prime}}\left(q_{i}^{\prime}\right)^{a_{i}^{\prime}}$, where $q_{i}, q_{i}^{\prime}$ are primes, $q_{i}>P$ and $q_{i}^{\prime} \leqslant P$ for each $i$, where $P=\frac{\sqrt{\log n}}{\log _{2} n}$. We have

$$
\omega(d(n)) \leqslant \omega\left(d\left(n_{1}\right)\right)+\omega\left(d\left(n_{2}\right)\right) .
$$

Since $\omega\left(n_{2}\right) \leqslant \pi(P) \ll \frac{\sqrt{\log n}}{\left(\log _{2} n\right)^{2}}$, Lemma 3.2 implies $\log d\left(n_{2}\right) \ll \sqrt{\log n} / \log _{2} n$. Applying the elementary inequality $\omega(u) \ll \frac{\log u}{\log _{2} u}$ gives

$$
\omega\left(d\left(n_{2}\right)\right) \ll \frac{\sqrt{\log n}}{\left(\log _{2} n\right)^{2}} .
$$

Next,

$$
\log n_{1} \geqslant(\log P) \sum_{i=1}^{r} a_{i}=\left(\frac{\log _{2} n}{2}-\log _{3} n\right) \sum_{i=1}^{r} a_{i} .
$$

Letting $s=\omega\left(d\left(n_{1}\right)\right)=\omega\left(\prod\left(a_{i}+1\right)\right)$, Lemma 3.4 implies that

$$
\sum_{i=1}^{r} a_{i} \geqslant \sum_{i=1}^{r} \sum_{p \mid\left(a_{i}+1\right)}(p-1) \geqslant \sum_{i=1}^{s}\left(p_{i}-1\right) \geqslant \sum_{i=1}^{s}(i \log i+O(1))=\frac{1}{2} s^{2} \log s+O\left(s^{2}\right) .
$$

Here we used the one-sided inequality $p_{i} \geqslant i \log i+O(1)$ deduced from (2.1). Thus,

$$
\log n \geqslant \log n_{1} \geqslant\left(\frac{1}{4}+O\left(\frac{\log _{3} n}{\log _{2} n}\right)\right)\left(\log _{2} n\right) s^{2} \log s+O\left(s^{2} \log _{2} n\right) .
$$

Consider two cases: (i) $s \leqslant \frac{\sqrt{\log n}}{\log _{2} n}$, (ii) $s>\frac{\sqrt{\log n}}{\log _{2} n}$. In case (ii), we have $\frac{\log n}{\log _{2}^{2} n} \geqslant\left(\frac{1}{8}+O\left(\frac{\log _{3} n}{\log _{2} n}\right)\right) s^{2}$, and we obtain in both cases

$$
\omega\left(d\left(n_{1}\right)\right)=s \leqslant \frac{\sqrt{8 \log n}}{\log _{2} n}+O\left(\frac{\sqrt{\log n} \log _{3} n}{\log _{2}^{2} n}\right),
$$

Combining this inequality with (4.1) and (4.2), we obtain the desired upper bound for $\omega(d(n))$.

Acknowledgement. The authors would like to thank Prof. L. G. Lucht for discussions on the subject and helpful comments on an earlier version of this paper.

\section{References}

[1] P. Erdős, Ramanujan and I. Number Theory, Madras 1987, Lecture Notes in Mathematics 1395, (Springer-Verlag, New York, 1989), 81-92.

[2] P. Erdős, A. Ivić, On the iterates of the enumerating function of finite abelian groups. Bull. Acad. Serbe Sci. Math. 17 (1989), 13-22.

[3] P. Erdős, I. Kátai, On the growth of $d_{k}(n)$. Fibonacci Quart. 7 (1969), 267-274.

[4] G. H. Hardy and E. M. Wright, An Introduction to the theory of numbers, Fifth Edition, The Clarendon Press, Oxford University Press, New York, 1979.

[5] A. Ivić, On the maximal order of certain arithmetic functions. Algebra, logic \& discrete mathematics (Niš, 1995). Filomat No. 9, part 3 (1995), 483-492.

[6] A. Ivić, Problem No. CNTA 5.3 in R. K. Guy, Conference Problems Session Conducted by J. L. Selfridge. Number theory (Ottawa, ON, 1996), CRM Proc. Lecture Notes, 19, Amer. Math. Soc., Providence, RI (1999), 385-390.

[7] S. Ramanujan, Highly composite numbers. Proc. London Math. Soc., series 2, 14 (1915), 347-409. Republished in Collected papers of Srinivasa Ramanujan, AMS Chelsea Publ., Providence, RI, 2000, p. 78-128. 
[8] A. Smati, Sur un problème de Ramanujan. C. R. Math. Acad. Sci. Paris 340 (2005), no. 1, 1-4.

[9] A. Smati, Sur un problème d'Erdôs et Kátai. Ann. Univ. Sci. Budapest. Sect. Comput. 29 (2008), 213-238.

[10] S. Wigert, Sur l'ordre grandeur du nombre de diviseurs d'un entier. Ark. Mat. 3, no. 18 (1907), 1-9.

Y. Buttkewitz: Department of Mathematics, Royal Holloway, University of London, Egham, SURREy TW20 OEX, U.K.

E-mail address: leroset-online.de

C. Elsholtz: Institut für Mathematik A, Technische Universität Graz, Steyrergasse 30, A-8010 Graz, Austria

E-mail address: elsholtz@math.tugraz.at

K. Ford: Department of Mathematics, 1409 West Green Street, UniVersity of Illinois At Urbana-Champaign, URBANA, IL 61801, USA

E-mail address: ford@math.uiuc.edu

J.-C. Schlage-Puchta: Department of Mathematics, Building S22, Ghent University, 9000 Gent, Belgium

E-mail address: jcsp@cage.ugent. be 